\title{
Discretization by Finite Elements of a Model Parameter Dependent Problem
}

\author{
Douglas N. Arnold \\ Department of Mathematics and Institute for Physical Science and Technology, \\ University of Maryland, College Park, Maryland 20742, USA
}

\begin{abstract}
The discretization by finite elements of a model variational problem for a clamped loaded beam is studied with emphasis on the effect of the beam thickness, which appears as a parameter in the problem, on the accuracy. It is shown that the approximation achieved by a standard finite element method degenerates for thin beams. In contrast a large family of mixed finite element methods are shown to yield quasioptimal approximation independent of the thickness parameter. The most useful of these methods may be realized by replacing the integrals appearing in the stiffness matrix of the standard method by Gauss quadratures.
\end{abstract}

Subject Classifications: AMS(MOS): 65 N30; CR: 5.17.

\section{Introduction}

In this paper we examine the finite element discretization of a model variational problem in which the dependent variables represent the vertical displacement and the rotation of the vertical fibers of a clamped loaded beam. The thickness of the beam appears parametrically in this model and we set as a goal to approximate the solution as accurately as possible for all values of this parameter.

In Sect. 2 some notations are collected and the beam model is presented in Sect. 3. In the following section we consider discretization by direct application of Galerkin's method with continuous piecewise polynomial subspaces of equal degree for the two variables. Although this standard finite element method produces quasioptimal approximation in $H^{1}$ and optimal order approximation in $L^{2}$, the approximation degenerates as the thickness of the beam decreases, resulting in a reduced uniform order of convergence in some norms, or, in the case of linear elements, in divergence. Thus the standard finite element method fails to achieve the goal set out above.

By introducing the shear stress as a third dependent variable one arrives at an equivalent variational formulation of the beam model. The discretization of 
this second variational principle, which is mixed in the sense that now both displacements and stresses are present, is the subject of Sect. 5. Specifically we show that for (almost) any choice of finite element spaces for the original displacement variables, there is a finite element space in which to approximate the shear stress variable such that the resulting mixed finite element method is stable, with stability constant independent of the beam thickness. Consequently quasioptimal estimates hold (for the triple of variables) without the degeneracy mentioned above. The proof of this result relies on an abstract stability theorem whose proof is deferred until Sect. 7 of the paper.

The additional stress variable introduced in the mixed formulation can be eliminated analytically after discretization, giving rise to what may be called an indirect displacement method. This method involves only the two original variables. When these are sought in continuous piecewise polynomial spaces of the same degree this indirect method achieves our goal of optimal order accuracy for thick and thin beams, and is thus superior to the direct displacement method. Moreover, in this case the indirect displacement method admits an interesting and convenient interpretation. As is shown in Sect. 6, it differs from the direct method only in that certain integrals are computed using a Gaussian quadrature rule rather than exactly. Thus it is an example of a reduced integration finite element method.

Reduced integration - the artifice of lowering the order of the integration rule used to compute certain contributions to the stiffness matrix in order to achieve better approximation - has received much attention in the engineering literature over the past decade $[5-10,12,13]$. Its principle success has been in thin plate problems (the two dimensional analogue of the problem considered here) and in modelling nearly incompressible materials. Numerous heuristic reasons for its success have been advanced, but no complete mathematical analysis exists. Bercovier [2] justifies one reduced integration triangular element for incompressible elasticity, although that approximation method does not converge with optimal order for the degree employed. The simpler one dimensional problem considered here allows a complete explanation.

In the final section of the paper some numerical results are presented comparing the error in the standard and reduced integration finite element approximations of the beam model. These decisively substantiate the theory presented.

\section{Notations}

All function spaces shall be formed with respect to the unit interval $I$. The $L^{2}$ inner product of two functions $f$ and $g$ is denoted $\langle f, g\rangle$. The associated norm of the function $f$ is written $\|f\|$, while $\|f\|_{r}$ denotes the norm in the Sobolev space $H^{r}$ :

$$
\|f\|_{r}^{2}=\|f\|^{2}+\left\|f^{\prime}\right\|^{2}+\ldots+\left\|f^{(r)}\right\|^{2} .
$$


On $H^{1}=\left\{f \in H^{1} \mid f(0)=f(1)=0\right\}$ the norm $f \rightarrow\left\|f^{\prime}\right\|$ is equivalent to the $H^{1}$ norm. For $g \in H^{-1}$, the space dual to $\dot{H}^{1}$, we thus set

$$
\|g\|_{-1}=\sup _{f \in \dot{H}^{1}}\langle f, g\rangle /\left\|f^{\prime}\right\| .
$$

Finally, we denote by $V$ the space $\stackrel{\circ}{H}^{1} \times \stackrel{H}{ }^{1}$ and use the norm $\|\varphi, \omega\|_{V}=\left(\left\|\varphi^{\prime}\right\|^{2}\right.$ $\left.+\left\|\omega^{\prime}\right\|^{2}\right)^{\frac{1}{2}}$.

For $0<d \leqq 1$ we introduce the inner product $B_{d}(\cdot, \cdot)$ on $V$ defined by

$$
B_{d}(\varphi, \omega ; \psi, v)=\left\langle\varphi^{\prime}, \psi^{\prime}\right\rangle+d^{-2}\left\langle\varphi-\omega^{\prime}, \psi-v^{\prime}\right\rangle
$$

for $(\varphi, \omega),(\psi, \nu) \in V$, and denote the associated norm

$$
\|\varphi, \omega\|_{d}=\left[B_{d}(\varphi, \omega ; \varphi, \omega)\right]^{\frac{1}{2}} .
$$

Note that by the triangle inequality and Poincarés inequality, $\left\|\omega^{\prime}\right\| \leqq\left\|\varphi-\omega^{\prime}\right\|$ $+\left\|\varphi^{\prime}\right\|$ for all $(\varphi, \omega) \in V$, so that

$$
\|\varphi, \omega\|_{V}^{2} \leqq 3\|\varphi, \omega\|_{d}^{2} \leqq\left(3+6 d^{-2}\right)\|\varphi, \omega\|_{V}^{2} .
$$

For the purposes of discretization we shall use finite element spaces defined with reference to partitions of $I$. If $\Delta=\left\{x_{0}, x_{1}, \ldots, x_{n}\right\}$ is a partition of $I$ ( 0 $\left.=x_{0}<\ldots<x_{n}=1\right)$, the maximum subinterval length is denoted $h_{\Delta}=\max \left(x_{i}\right.$ $-x_{i_{-1}}$ ). We shall deal only with quasiuniform partitions. That is, the phrase "for all $\Delta$ ", explicit or implied, refers to all partitions $\Delta$ satisfying

$$
\alpha \min \left(x_{i}-x_{i-1}\right) \geqq h_{A}
$$

with $\alpha$ being a fixed constant.

For $r \geqq 0$ and a partition $\Delta, \mathscr{M}_{-1}^{r}(\Delta)$ denotes the space of functions on $I$ which restrict to polynomial functions of degree at most $r$ on each subinterval $\left(x_{i-1}, x_{i}\right)$. The subscript -1 refers to the lack of continuity constraint. For $k \geqq 0$ we let $\mathscr{M}_{k}^{r}(\Delta)=\mathscr{M}_{-1}^{r}(\Delta) \cap C^{k}(I)$ and $\mathscr{H}_{k}^{r}(\Delta)=\mathscr{M}_{k}^{r}(\Delta) \cap \mathscr{H}^{1}$. Finally we define $V^{r}(\Delta)=\mathscr{U}_{0}^{r}(\Delta) \times \mathscr{M}_{0}^{r}(\Delta)$.

The letter $C$ is used to denote a generic constant independent of $d \in(0,1]$ and the partition $\Delta$.

\section{The Beam Model}

The model variational problem we consider is

$$
\begin{aligned}
& \text { find }\left(\varphi_{d}, \omega_{d}\right) \in V \text { such that } \\
& \left\langle\varphi_{d}^{\prime}, \psi^{\prime}\right\rangle+a_{1} d^{-2}\left\langle\varphi_{d}-\omega_{d}^{\prime}, \psi-v^{\prime}\right\rangle=a_{2}\langle g, v\rangle \quad \text { for all }(\psi, v) \in V .
\end{aligned}
$$

Here $a_{1}$ and $a_{2}$ are positive constants. For convenience and without loss of generality we shall assume that $a_{1}=a_{2}=1$ so the quantity appearing on the left hand side is simply $B_{d}\left(\varphi_{d}, \omega_{d} ; \psi, v\right)$. The boundary value problem associated 
with $\left(S_{d}\right)$ states that

$$
\begin{gathered}
-\varphi_{d}^{\prime \prime}+d^{-2}\left(\varphi_{d}-\omega_{d}^{\prime}\right)=0 \quad \text { on } I, \\
d^{-2}\left(\varphi_{d}-\omega_{d}^{\prime}\right)^{\prime}=g \quad \text { on } I, \\
\varphi_{d}(0)=\varphi_{d}(1)=\omega_{d}(0)=\omega_{d}(1)=0 .
\end{gathered}
$$

This system, which was studied by Timoshenko [11], may be derived from the classical theory of plane linear elasticity by dimensional reduction. Briefly, we suppose that the undisplaced beam occupies the region $I \times[-d / 2, d / 2]$ in the $(x, y)$-plane and is subject to a vertical body force $-d^{2} g(x)$ which is independent of $y$. (The factor $d^{2}$ assures that the energy has a finite, non-zero limit as $d \rightarrow 0$.) Letting $u$ and $v$ denote the horizontal and vertical displacements, the energy of the beam is

$$
E=\frac{1}{2} \int_{0}^{1} \int_{-\frac{d}{2}}^{\frac{d}{2}}\left\{\lambda\left(u_{x}+v_{y}\right)^{2}+2 \mu\left[u_{x}^{2}+v_{y}^{2}+\left(u_{y}+v_{x}\right)^{2} / 2\right]-2 d^{2} g v\right\} d y d x,
$$

where $\lambda$ and $\mu$ are the Lamé coefficients. This quantity is to be minimized over the set of displacements satisfying $u=v=0$ when $x \in\{0,1\}$. Prior to minimization, however, we impose Mindlin's hypotheses:

1) the vertical displacement is independent of $y$;

2) the midline $y=0$ is not displaced horizontally;

3) the vertical fibers $x=$ constant remain linear after displacement.

Mathematically this requires that $u$ and $y$ have the special form

$$
\begin{aligned}
& u(x, y)=-y \varphi_{d}(x), \\
& v(x, y)=\omega_{d}(x),
\end{aligned}
$$

where $\varphi_{d}, \omega_{d} \in H^{1}$; although using symmetry one can show that the formally weaker assumption that $u$ and $v$ are linear in $y$ leads to the same conclusion. By minimizing $E$ over all such $(u, v)$ we arrive at problem $\left(S_{d}\right)$ (with $a_{2}=12 /(\lambda$ $+2 \mu), a_{1}=a_{2} \mu$ ).

When $d \ll 1$ one often assumes in addition to Mindlin's hypotheses the Kirchoff hypothesis that the vertical fibers remain normal, i.e., that $\varphi_{d}=\omega_{d}^{\prime}$. Energy minimization then leads to a one dimensional biharmonic problem for the vertical displacement. The model considered here is often viewed as a penalty procedure for that problem. However, since the Timoshenko model is less restrictive our concern is the direct approximation of $\left(S_{d}\right)$ for $d \in(0,1]$.

Before considering discretization we prove a theorem asserting the wellposedness and regularity of solutions for a slightly more general problem than $\left(S_{d}\right)$.

Theorem 3.1. Let $f, g \in H^{-1}, 0<d \leqq 1$. Then there exists a unique pair of functions $(\rho, \mu) \in V$ such that

$$
B_{d}(\rho, \mu ; \psi, v)=\langle f, \psi\rangle+\langle g, v\rangle \quad \text { for all }(\psi, v) \in V .
$$


Moreover, for $p=0,1, \ldots$, there is a constant $C$ depending only on $p$ such that

$$
\|\rho\|_{p+1}+\|\mu\|_{p+1}+d^{-2}\left\|\rho-\mu^{\prime}\right\|_{p} \leqq C\left(\|f\|_{p-1}+\|g\|_{p-1}\right) .
$$

Proof. The existence of a unique solution is guaranteed by the Riesz representation theorem. Setting $(\psi, v)=(0, \mu)$ in $(3.1)$ we see that

$$
\left\|\mu^{\prime}\right\| \leqq\|\rho\|+d^{2}\|g\|_{-1} .
$$

The choice $(\psi, v)=(\rho, 0)$ then gives

and so

$$
\left\|\rho^{\prime}\right\| \leqq\|f\|_{-1}+\|g\|_{-1},
$$

$$
\left\|\mu^{\prime}\right\| \leqq\|f\|_{--1}+\left(1+d^{2}\right)\|g\|_{-1} .
$$

Next take $(\psi, v)= \pm\left(\left(x-x^{2}\right) / 2,\left(-2 x^{3}+3 x^{2}-x\right) / 12\right)$. It follows that

$$
|\langle\rho, 1\rangle| \leqq C d^{2}\left(\|f\|_{-1}+\|g\|_{-1}\right) .
$$

Finally, setting $\psi=0, v(x)=\mu(x)-\int_{0}^{x}[\rho-\langle\rho, 1\rangle]$, we get

$$
\begin{aligned}
\left\|\rho-\mu^{\prime}\right\|^{2} & =\langle\rho, 1\rangle^{2}+d^{2}\langle g, v\rangle \\
& \leqq\langle\rho, 1\rangle^{2}+d^{2}\|g\|_{-1}\left[\left\|\rho-\mu^{\prime}\right\|+|\langle\rho, 1\rangle|\right],
\end{aligned}
$$

and hence

$$
\left\|\rho-\mu^{\prime}\right\| \leqq C d^{2}\left(\|f\|_{-1}+\|g\|_{-1}\right) .
$$

This proves (3.2) when $p=0$. The estimate for $p \geqq 1$ follows by differentiating the differential equations satisfied by $\rho$ and $\mu$ and employing a simple induction.

\section{The Standard Finite Element Method}

Let $r \geqq 1$ and let $\Delta$ be a partition. An obvious discretization of $\left(S_{d}\right)$ results from employing Galerkin's method with the subspace $V^{r}(\Delta)$ of $V$.

$$
\begin{aligned}
& \text { Find }\left(\varphi_{d \Delta}, \omega_{d \Delta}\right) \in V^{r}(\Delta) \text { such that } \\
& B_{d}\left(\varphi_{d \Delta}, \omega_{d \Delta} ; \psi, v\right)=\langle g, v\rangle \text { for all }(\psi, v) \in V^{r}(\Delta) .
\end{aligned}
$$

It is easy to prove that for fixed $d, \varphi_{d \Delta}$ and $\omega_{d \Delta}$ converge to $\varphi_{d}$ and $\omega_{d}$ in both $L^{2}$ and $H^{1}$ at the approximation theoretic optimal rate with respect to $h_{A}$. Such convergence however is not uniform with respect to the thickness $d$. The following theorem gives the rates of convergence which hold both for constant $d$ and uniform in $d$.

Theorem 4.1. Let $\left(\varphi_{d}, \omega_{d}\right) \in V$ and $\left(\varphi_{d \Delta}, \omega_{d A}\right) \in V^{r}(\Delta)$ solve $\left(S_{d}\right)$ and $\left(S_{d \Delta}\right)$ respectively. Denote by $\alpha_{d \Delta}=\varphi_{d}-\varphi_{d \Delta}$ and $\beta_{d \Delta}=\omega_{d}-\omega_{d \Delta}$ the errors. Then there exists a constant $C$ independent of $d \in(0,1], g \in H^{r-1}$, and the partition $\Delta$ such that 


$$
\begin{aligned}
& \left\|\alpha_{d \Delta}\right\| \leqq \begin{cases}C \min \left(d^{-2} h^{2}, 1\right)\|g\| & \text { if } r=1, \\
C \min \left(d^{-1} h^{r+1}, h^{r}\right)\|g\|_{r-1} & \text { if } r>1,\end{cases} \\
& \left\|\alpha_{d \Delta}^{\prime}\right\| \leqq C \min \left(d^{-1} h^{r}, h^{r-1}\right)\|g\|_{r-1}, \\
& \left\|\beta_{d \Delta}\right\| \leqq \begin{cases}C \min \left(d^{-2} h^{2}, 1\right)\|g\| & \text { if } r=1, \\
C \min \left(d^{-1} h^{r+1}, h^{r}\right)\|g\|_{r-1} & \text { if } r>1,\end{cases} \\
& \left\|\beta_{d \Delta}^{\prime}\right\| \leqq \begin{cases}C \max \left[h, \min \left(d^{-2} h^{2}, 1\right)\right]\|g\| & \text { if } r=1, \\
C h^{r}\|g\|_{r-1} & \text { if } r>1 .\end{cases}
\end{aligned}
$$

Proof. We first establish the auxilliary estimate

$$
\left\|\alpha_{d \Delta}, \beta_{d \Delta}\right\|\left\|_{d} \leqq C \min \left(d^{-1} h^{r}, h^{r-1}\right)\right\| g \|_{r \ldots 1} .
$$

Since $\left(\varphi_{d d}, \omega_{d A}\right)$ is determined by projection in a Hilbert space,

Consequently

$$
\left\|\alpha_{d d}, \beta_{d \Delta}\right\|_{d}=\inf _{(\varphi, \omega) \in \mathcal{V}^{r}(\Delta)}\left\|\varphi_{d}-\varphi, \omega_{d}-\omega\right\|_{d} .
$$

$$
\begin{aligned}
\left\|\alpha_{d A}, \beta_{d A}\right\| \|_{d} & \leqq C d^{-1} \inf _{(\varphi, \omega) \in V^{r}(\Delta)}\left\|\varphi_{d}-\varphi, \omega_{d}-\omega\right\|_{V} \\
& \leqq C d^{-1} h^{r}\left(\left\|\varphi_{d}\right\|_{r+1}+\left\|\omega_{d}\right\|_{r+1}\right) \\
& \leqq C d^{-1} h^{r}\|g\|_{r-1}
\end{aligned}
$$

Since $\left\|\alpha_{d \Delta}, \beta_{d \Delta}\right\|_{d}$ is clearly bounded independent of $d$ and $\Delta$, this proves (4.5) in case $r=1$. If $r>1$, let $\varphi \in \mathscr{M}_{0}^{r-1}(\Delta)$ satisfy $\left\|\varphi_{d}-\varphi\right\|_{1} \leqq C h^{r-1}\|g\|_{r-1}$ and $\left\langle\varphi_{d}\right.$ $-\varphi, 1\rangle=0$. Set $\rho=\varphi-\varphi_{d}+\omega_{d}^{\prime}$. Then $\langle\rho, 1\rangle=0$, so there exists $v \in \mathscr{M}_{0}^{r-1}(\Delta)$ such that $\langle v, 1\rangle=0$ and

$$
\|v-\rho\| \leqq C h^{r}\left\|\rho^{(r)}\right\|=C h^{r}\left\|\left(\varphi_{d}-\omega_{d}^{\prime}\right)^{(r)}\right\| \leqq C d h^{r}\|g\|_{r-1} .
$$

Here $\rho^{(r)}$ denotes the $r$ th derivative of $\rho$ taken piecewise. The former condition assures that $v$ is the derivative of a function $\omega$ in $\mathscr{M}_{1}^{r}(\Delta)$, and then

$$
\begin{aligned}
\left\|\alpha_{d \Delta}, \beta_{d \Delta}\right\|_{d}^{2} & \leqq\left\|\varphi_{d}-\varphi, \omega_{d}-\varphi\right\|_{d}^{2} \\
& =\left\|\varphi_{d}^{\prime}-\varphi^{\prime}\right\| l^{2}+d^{-2}\|v-\rho\|^{2} \leqq C h^{2 r-2}\|g\|_{r-1}^{2} .
\end{aligned}
$$

This completes the proof of (4.5). The estimate (4.2) is an immediate consequence.

To prove (4.1) we use a duality argument. Define $(\rho, \mu) \in V$ by the equations

$$
B_{d}(\psi, v ; \rho, \mu)=\left\langle\psi, \alpha_{d \Delta}\right\rangle \quad \text { for all }(\psi, v) \in V .
$$

Then, by Theorem 3.1, $\left\|\rho^{\prime \prime}\right\|+\left\|\mu^{\prime \prime}\right\| \leqq C\left\|\alpha_{d \Delta}\right\|$ and clearly $\rho-\mu^{\prime}$ is constant. Now

$$
\begin{aligned}
\left\|\alpha_{d \Delta}\right\|^{2} & =B_{d}\left(\alpha_{d \Delta}, \beta_{d \Delta} ; \rho, \mu\right) \\
& =B_{d}\left(\alpha_{d \Delta}, \beta_{d \Delta} ; \rho-\psi, \mu-v\right) \\
& \leqq\left\|\alpha_{d \Delta}^{\prime}\right\|\left\|\rho^{\prime}-\psi^{\prime}\right\|+d^{-2}\left\|\alpha_{d \Delta}-\beta_{d \Delta}^{\prime}\right\|\left\|\rho-\psi-\mu^{\prime}+v^{\prime}\right\| \\
& \text { for all }(\psi, v) \in V^{r}(\Delta) .
\end{aligned}
$$


Let $\psi \in \mathscr{\mathscr { M }}_{0}^{1}(\Delta)$ be chosen such that $\left\|\psi^{\prime}\right\| \leqq C\left\|\rho^{\prime}\right\|,\left\|\rho^{\prime}-\psi^{\prime}\right\| \leqq C h\left\|\rho^{\prime \prime}\right\| \leqq C h\left\|\alpha_{d \alpha}\right\|$, and $\langle\rho-\psi, 1\rangle=0$. Then $\rho-\psi-\mu^{\prime} \in \mathscr{M}_{0}^{1}(\Delta)$ and $\left\langle\rho-\psi-\mu^{\prime}, 1\right\rangle=0$. In the case of linear elements let $\omega \in \mathscr{M}_{-1}^{0}(\Delta)$ interpolate $\rho-\psi-\mu^{\prime}$ at the midpoints of each subinterval of $\Delta$. Then $v(x)=\int_{0}^{x} \omega$ determines a function in $\mathscr{\mathscr { M }}_{0}^{1}(\Delta)$ and

$$
\begin{aligned}
\left\|\rho-\psi-\mu^{\prime}+v^{\prime}\right\| & \leqq C h\left\|\left(\rho-\psi-\mu^{\prime}\right)^{\prime}\right\|=C h\left\|\psi^{\prime}\right\| \\
& \leqq C h\left\|\rho^{\prime}\right\| \leqq C h\left\|\alpha_{d \Delta}\right\| .
\end{aligned}
$$

Therefore, from (4.6)

$$
\begin{aligned}
\left\|\alpha_{d \Delta}\right\|^{2} & \leqq C h\left(\left\|\alpha_{d \Delta}^{\prime}\right\|+d^{-2}\left\|\alpha_{d \Delta}-\beta_{d \Delta}^{\prime}\right\|\right)\left\|\alpha_{d \Delta}\right\| \\
& \leqq C h d^{-1}\left\|\alpha_{d \Delta}, \beta_{d \Delta}\right\|\left\|_{d}\right\| \alpha_{d \Delta} \|,
\end{aligned}
$$

and so

$$
\left\|\alpha_{d \Delta}\right\| \leqq C h d^{-1}\left\|\alpha_{d \Delta}, \beta_{d \Delta} \mid\right\|_{d} \leqq C d^{-2} h^{2}\|g\|,
$$

by (4.5). Since also $\left\|\alpha_{d \Delta}\right\| \leqq\left\|\alpha_{d \Delta}^{\prime}\right\|_{x}=C,(4.1)$ is established when $r=1$.

If $r>1$, we simply let $v(x)=\int_{0}^{x}\left(\rho-\psi-\mu^{\prime}\right)$. Then $v \in \mathscr{M}_{1}^{2}(\Delta)$. Applying (4.6) and (4.2) we get

$$
\left\|\alpha_{d \Delta}\right\|_{i}=C h\left\|\alpha_{d \Delta}^{\prime}\right\| \leqq C \min \left(d^{-1} h^{r+1}, h^{r}\right)\|g\|_{r-1} .
$$

We proceed next to the proof of (4.4). For all $v \in \mathscr{M}_{0}^{r}(\Delta)$ the pair $\left(0,-\beta_{d \Delta}+\omega_{d}\right.$ $-v) \in V^{r}(\Delta)$ and so the inner product $\left\langle\alpha_{d \Delta}-\beta_{d A}^{\prime},-\beta_{d \Delta}^{\prime}+\omega_{d}^{\prime}-v^{\prime}\right\rangle=0$. It follows that

$$
\left\|\beta_{d \Delta}^{\prime}\right\|^{2} \leqq\left(\left\|\alpha_{d \Delta}\right\|+\left\|\omega_{d}^{\prime}-v^{\prime}\right\|\right)\left\|\beta_{d \Delta}^{\prime}\right\|+\left\|\alpha_{d \Delta}\right\|\left\|\omega_{d}^{\prime}-v^{\prime}\right\|,
$$

and hence that

$$
\begin{aligned}
\left\|\beta_{d \Delta}^{\prime}\right\| & \leqq C\left(\left\|\alpha_{d \Delta}\right\|+\inf _{v \in \dot{H}_{0}^{\prime}(\Delta)}\left\|\omega_{d}^{\prime}-v^{\prime}\right\|\right) \\
& \leqq C\left(\left\|\alpha_{d \Delta}\right\|+h^{r}\|g\|_{r-1}\right) .
\end{aligned}
$$

Combining this estimate with (4.1) gives (4.4).

Finally we prove (4.3), again employing a duality argument. In this case, $(\rho, \mu) \in V$ is defined by

$$
B_{d}(\psi, v ; \rho, \mu)=\left\langle v, \beta_{d A}\right\rangle \quad \text { for all }(\psi, v) \in V^{r}(\Delta) .
$$

For $r=1$, we let $\left(\rho_{\Delta}, \mu_{A}\right) \in V^{1}(\Delta)$ be the projection of $(\rho, \mu)$ with respect to the inner product $B_{d}$. Then

$$
\begin{aligned}
\left\|\beta_{d \Delta}\right\|^{2} & =B_{d}\left(\alpha_{d \Delta}, \beta_{d \Delta} ; \rho, \mu\right) \\
& =B_{d}\left(\alpha_{d \Delta}, \beta_{d \Delta} ; \rho-\rho_{\Delta}, \mu-\mu_{\Delta}\right) \\
& \leqq\left\|\alpha_{d \Delta}, \beta_{d \Delta}\right\|_{d}\left\|\rho-\rho_{\Delta}, \mu-\mu_{\Delta}\right\|_{d} \\
& \leqq C \min \left(d^{-2} h^{2}, 1\right)\|g\|\left\|\beta_{d \Delta}\right\|,
\end{aligned}
$$

where we have employed (4.5) twice. 
For elements of higher order, we let $\left(\rho_{\Delta}, \mu_{\Delta}\right)$ be the $B_{d}$-projection of $(\rho, \mu)$ on $V^{2}(4)$. Then similar reasoning gives

$$
\begin{aligned}
\left\|\beta_{d \Delta}\right\|^{2} & \leqq\left\|\alpha_{d \Delta}, \beta_{d \Delta}\right\|\left\|_{d}\right\| \rho-\rho_{\Delta}, \mu-\mu_{\Delta} \|_{d} \\
& \leqq C \min \left(d^{-1} h^{r}, h^{r-1}\right)\|g\|_{r-1} \min \left(d^{-1} h^{2}, h\right)\left\|\beta_{d \Delta}\right\|_{1},
\end{aligned}
$$

which combined with (4.4) implies (4.3).

The following table summarizes the rates of convergence guaranteed by Theorem 4.1 .

Table 1. $L^{2}$ rates of convergence

\begin{tabular}{llllll}
\hline & & $\alpha_{d \Delta}$ & $\alpha_{d \Delta}^{\prime}$ & $\beta_{d \Delta}$ & $\beta_{d \Delta}^{\prime}$ \\
\hline$r=1$ & Fixed $d$ & 2 & 1 & 2 & 1 \\
& Uniform in $d$ & 0 & 0 & 0 & 0 \\
$r>1$ & Fixed $d$ & $r+1$ & $r$ & $r+1$ & $r$ \\
& Uniform in $d$ & $r$ & $r-1$ & $r$ & $r$ \\
\hline
\end{tabular}

Thus none of the quantities $\left\|\alpha_{d \Delta}\right\|,\left\|\alpha_{d \Delta}^{\prime}\right\|$, and $\left\|\beta_{d \Delta}\right\|$ is shown to converge to zero at the optimal rate uniformly in $d$, and, in the case $r=1$, neither is $\left\|\beta_{d A}^{\prime}\right\|$. We now prove that the uniform rates listed in the table are in fact sharp in each case, with one exception. Although numerical experiments such as those presented below in Sect. 8 support the claim that $\left\|\beta_{d \Delta}\right\|=O\left(h_{\Delta}^{r}\right)$ uniformly in $d$ when $r>1$, the author does not know how to prove that it is no smaller.

The following theorem states that uniform convergence of $\varphi_{d \Delta}$ in either $L^{2}$ or $H^{1}$ at a rate faster than predicted is impossible except in the trivial case when the solution functions are polynomials in the finite element space. The notation is as in Theorem 4.1 .

Theorem 4.2. Suppose that either

or

$$
\left\|\alpha_{d \Delta}\right\|=o\left(h_{\Delta}^{r}\right) \quad \text { uniformly in } d
$$

$$
\left\|\alpha_{d \Delta}^{\prime}\right\|=o\left(h_{\Delta}^{r-1}\right) \quad \text { uniformly in } d .
$$

Then $\varphi_{d}$ and $\omega_{d}$ are polynomials of degree at most $r$.

Proof. By applying an inverse property one may deduce from either (4.7) or (4.8) that

$$
\left\|\varphi_{d}^{(r)}-\varphi_{d \Delta}^{(r)}\right\|=o(1) \quad \text { uniformly in } d
$$

where $\varphi_{d \Delta}^{(r)}$ denotes the piecewise $r$ th derivative of $\varphi_{d A}$. We shall infer the conclusion of the theorem from (4.9).

By Theorem 3.1, $\omega_{d}$ is bounded in $H^{1}$ independent of $d$ and so

$$
\begin{aligned}
\left\|\varphi_{d \Delta}-\omega_{d \Delta}^{\prime}\right\|^{2} & \leqq d^{2}\left\|\varphi_{d \Delta}, \omega_{d \Delta}\right\|\left\|_{d}^{2} \leqq d^{2}\right\| \varphi_{d}, \omega_{d} \|_{d}^{2} \\
& =d^{2}\left\langle g, \omega_{d}\right\rangle \leqq C d^{2}
\end{aligned}
$$


where the constant $C$ depends only on $g$. Now since $\varphi_{d A}$ is a piecewise polynomial of degree $r$ and $\omega_{d A}^{\prime}$ is a piecewise polynomial of degree $r-1$, it follows that

$$
C\left\|\varphi_{d \Delta}-\omega_{d \Delta}^{\prime}\right\|^{2} \geqq h_{\Delta}^{2 r}\left\|\varphi_{d \Delta}^{(r)}\right\|^{2},
$$

$C$ depending only on $r$. Thus $\left\|\varphi_{d \Delta}^{(r)}\right\| \leqq C d h_{\Delta}^{-r}$ and consequently $\left\|\varphi_{d \Delta}^{(r)}\right\| \rightarrow 0$ for any sequence of pairs $(d, \Delta)$ satisfying $d+h_{A}+d h_{\Delta}^{-r} \rightarrow 0$. Combining this with (4.9) shows that $\left\|\varphi_{d}^{(r)}\right\| \rightarrow 0$ also. If $r \leqq 3$ this implies that $g=0$ since $g=\varphi_{d}^{\prime \prime \prime}$. For $r>3$ we get at least that $g^{(r-3)}=0$, and so $g$ is a polynomial of degree at most $r$ -4. It follows that $\varphi_{d}$ and $\omega_{d}$ are polynomials of degree at most $r-1$ and $r$ respectively.

In the case of linear elements the above proof shows that if $d$ tends to zero faster than $h_{\Delta}$, then $\varphi_{d \Delta}$ converges to zero in $H^{1}$. In light of (4.10) $\omega_{d \Delta}$ also converges to zero. Thus it is impossible that either $\varphi_{d \Delta}$ converges to $\varphi_{d}$ in $L^{2}$ uniformly in $d$ or that $\omega_{d \Delta}$ converges to $\omega_{d}$ in $L^{2}$ uniformly in $d$, except in the trivial case when $\varphi_{d \Delta}=\omega_{d \Delta}=0$.

\section{A Mixed Variational Principle}

Let $\zeta_{d}=d^{-2}\left(\varphi_{d}-\omega_{d}^{\prime}\right)$. Then, by Theorem 3.1, $\zeta_{d} \in L^{2}$ and $\left\|\zeta_{d}\right\| \leqq C\|g\|_{-1}$ with $C$ independent of $d$. The standard variational problem $\left(S_{d}\right)$ of Sect. 3 (with $a_{1}=a_{2}$ $=1$ ) is equivalent to the mixed variational system

$$
\begin{aligned}
& \text { find }\left(\varphi_{d}, \omega_{d}, \zeta_{d}\right) \in V \times L^{2} \text { such that } \\
& \left\langle\varphi_{d}^{\prime}, \psi^{\prime}\right\rangle+\left\langle\zeta_{d}, \psi-v^{\prime}\right\rangle=\langle g, v\rangle \quad \text { for all }(\psi, v) \in V, \\
& \left\langle\varphi_{d}-\omega_{d}^{\prime}, \eta\right\rangle-d^{2}\left\langle\zeta_{d}, \eta\right\rangle=0 \quad \text { for all } \eta \in L^{2} .
\end{aligned}
$$

If $d$ is set to zero this system becomes of the form studied by Brezzi [3], Falk and Osborn [4], and others. For positive $d,\left(M_{d}\right)$ is of the general form considered by Bercovier [2], but his results concern the approximation of the limiting problem $(d=0)$ and so cannot be used to derive the uniform approximation results we desire. We shall instead employ the following abstract stability theorem which will be proved in Sect. 7 .

Theorem 5.1. Let $\mathscr{V}$ and $\mathscr{W}$ be Hilbert spaces, $a: \mathscr{V} \times \mathscr{V} \rightarrow \mathbb{R}$ and $b: \mathscr{V} \times \mathscr{W} \rightarrow \mathbb{R}$ bounded bilinear forms, and $d \in[0,1]$. Suppose that the following conditions are fulfilled:

1) $a$ is symmetric and positive semidefinite;

2) there exists $C_{1}>0$ such that for all $z \in \mathscr{Z}=\{v \in \mathscr{V} \mid b(v, w)=0$ for all $w \in$ $\mathscr{W}\}$,

$$
C_{1} a(z, z) \geqq\|z\|_{\mathscr{x}}^{2} ;
$$

3) there exists $C_{2}>0$ such that for all $w \in \mathscr{W}$ there exists $v \in \mathscr{V}$ with

$$
C_{2} b(v, w) \geqq\|v\|_{\mathscr{r}}\|w\|_{\mathscr{w}} .
$$

Then, for each pair $(f, g) \in \mathscr{V} \times \mathscr{W}$ there exists a unique pair $(v, w) \in \mathscr{V} \times \mathscr{W}$ such that

$$
a(v, u)+b(u, w)=\langle f, u\rangle_{\mathscr{V}} \quad \text { for all } u \in \mathscr{V}
$$


and

$$
b(v, y)-d^{2}\langle w, y\rangle_{\mathscr{W}}=\langle g, y\rangle_{\mathscr{W}} \quad \text { for all } y \in \mathscr{W} \text {. }
$$

Moreover $\|v\|_{\mathscr{V}}+\|w\|_{\mathscr{W}} \leqq C\left(\|f\|_{\mathscr{V}}+\|g\|_{\mathscr{W}}\right)$, the constant $C$ depending on $C_{1}$, $C_{2}$, and the norms of the bilinear forms $a$ and $b$, but not on $d$.

We now show that the variational system $\left(M_{d}\right)$ fulfills the hypotheses of Theorem 5.1. Here $\mathscr{V}=V, \mathscr{W}=L^{2}$,

and

$$
a(\varphi, \omega ; \psi, v)=\left\langle\varphi^{\prime}, \psi^{\prime}\right\rangle,
$$

$$
b(\varphi, \omega ; \zeta)=\left\langle\varphi-\omega^{\prime}, \zeta\right\rangle,
$$

for $(\varphi, \omega),(\psi, v) \in V, \zeta \in L^{2}$. Indeed $a$ is clearly semidefinite over $\mathscr{V}$. In this case the kernal space $\mathscr{Z}=\left\{(\varphi, \omega) \mid \omega \in \check{H}^{2}, \varphi=\omega^{\prime}\right\}$. Hence, for $(\varphi, \omega) \in \mathscr{Z}$,

$$
a(\varphi, \omega ; \varphi, \omega)=\left\|\varphi^{\prime}\right\|^{2} \geqq \frac{1}{2}\left(\left\|\varphi^{\prime}\right\|^{2}+\|\varphi\|^{2}\right)=\frac{1}{2}\left(\left\|\varphi^{\prime}\right\|^{2}+\left\|\omega^{\prime}\right\|^{2}\right),
$$

which verifies the second hypothesis. To verify the third, we may set, for given $\zeta \in L^{2}, \varphi(x)=6\langle\zeta, 1\rangle\left(x-x^{2}\right)$ and define $\omega \in \dot{H}^{1}$ by $\omega^{\prime}=\varphi-\zeta$.

Now let $\mathscr{M}$ and $\mathscr{N}$ denote finite dimensional subspaces of $H^{1}$ and consider the approximation of $\left(M_{d}\right)$ via Galerkin's method using $\mathscr{V}=\mathscr{M} \times \mathscr{N}$ to approximate $V$ and

$$
\mathscr{W}=\left\{\omega^{\prime}+c \mid \omega \in \mathscr{N}, c \in \mathbb{R}\right\}
$$

to approximate $L^{2}$. We assume that $\mathscr{M}$ contains elements of non-zero mean value (which is certainly necessary if $\mathscr{M}$ is to approximate $H^{1}$ well), and set $C_{0}$ $=\inf \left\{\left\|\varphi^{\prime}\right\| \mid \varphi \in \mathscr{M},\langle\varphi, 1\rangle=1\right\}$. (For common finite element spaces $C_{0}<6$.) With this choice of spaces we verify that the hypotheses of Theorem 5.1 remain valid. The first hypothesis is again obvious. For the second we identify $\mathscr{Z}$ $=\left\{(\varphi, \omega) \in \mathscr{V} \mid \omega^{\prime}=\pi_{\mathscr{W}} \varphi\right\}$ where $\pi_{\mathscr{W}}$ is the $L^{2}$ projection onto $\mathscr{W}$. Therefore if $(\varphi, \omega) \in \mathscr{Z}, \quad a(\varphi, \omega ; \varphi, \omega) \geqq \frac{1}{2}\left(\left\|\varphi^{\prime}\right\|^{2}+\left\|\omega^{\prime}\right\|^{2}\right)$. Finally we verify the third hypothesis of the theorem. Fix $\varphi_{0} \in \mathscr{M}$ with $\left\langle\varphi_{0}, 1\right\rangle=1$ and $\left\|\varphi_{0}^{\prime}\right\| \leqq C_{0}$. Given $\zeta \in$ $\mathscr{W}$ set $\varphi=\langle\zeta, 1\rangle \varphi_{0}$. Then $\pi_{\mathscr{W}} \varphi-\zeta \in \mathscr{W}$ and $\left\langle\pi_{\mathscr{W}} \varphi-\zeta, 1\right\rangle=0$, so there exists $\omega \in \mathscr{N}$ satisfying $\omega^{\prime}=\pi_{\mathscr{W}} \varphi-\zeta$. Moreover, $\left\|\varphi^{\prime}\right\|=C_{0}\|\zeta\|,\left\|\omega^{\prime}\right\| \leqq\left(1+C_{0}\right)\|\zeta\|$, and $b(\varphi, \omega ; \zeta)=\|\zeta\|^{2}$, yielding hypothesis 3 ) with $C_{2}$ depending only on $C_{0}$. From the theorem we conclude that the mapping from $\mathscr{V} \times \mathscr{W}$ to itself associated with the Galerkin equations is an isomorphism. It follows in a standard way [1] that the Galerkin solution is quasioptimal. This is summarized in the following theorems.

Theorem 5.2. Let $\mathscr{M}$ and $\mathscr{N}$ be finite dimensional subspaces of $\dot{H}^{1}$ and assume that there exists $\varphi \in \mathscr{M}$ with $\langle\varphi, 1\rangle=1$ and $\left\|\varphi^{\prime}\right\| \leqq C_{0}$. Set $\mathscr{W}=\left\{\omega^{\prime}\right.$ $+c \mid \omega \in \mathcal{N}, c \in \mathbb{R}\}$. Then for $d \in[0,1]$ there exists a unique triple $\left(\varphi_{d, \mathscr{M}}, \omega_{d \mathscr{N}}, \zeta_{d \mathscr{W}}\right) \in \mathscr{M} \times \mathscr{N} \times \mathscr{W}$ such that

$$
\begin{array}{ll}
\left\langle\varphi_{d \mathscr{M}}^{\prime}, \psi^{\prime}\right\rangle+\left\langle\zeta_{d \mathscr{W}}, \psi-v^{\prime}\right\rangle=\langle g, v\rangle & \text { for all }(\psi, v) \in \mathscr{M} \times \mathscr{N}, \\
\left\langle\varphi_{d \mathscr{M}}-\omega_{d \mathscr{N}}^{\prime}, \eta\right\rangle-d^{2}\left\langle\zeta_{d \mathscr{W}}, \eta\right\rangle=0 & \text { for all } \eta \in \mathscr{W} .
\end{array}
$$


Moreover there exists a constant $C$ independent of $d$ and depending on $\mathscr{M}$ and $\mathscr{N}$ only through $C_{0}$ such that

$$
\begin{aligned}
& \left\|\varphi_{d}^{\prime}-\varphi_{d \cdot \mathcal{M}}^{\prime}\right\|+\left\|\omega_{d}^{\prime}-\omega_{d \mathcal{N}}^{\prime}\right\|+\left\|\zeta_{d}-\zeta_{d \mathscr{W}}\right\| \\
& \quad \leqq C \inf \left\{\left\|\varphi_{d}^{\prime}-\varphi^{\prime}\right\|+\left\|\omega_{d}^{\prime}-\omega^{\prime}\right\|+\left\|\zeta_{d}-\zeta\right\| \|(\varphi, \omega, \zeta) \in \mathscr{M} \times \mathscr{N} \times \mathscr{W}\right\} .
\end{aligned}
$$

Note that if $d \neq 0$, then

$$
\zeta_{d \mathscr{W}}=d^{-2} \pi_{\mathscr{W}}\left(\varphi_{d \cdot \mathscr{H}}-\omega_{d \mathscr{N}}^{\prime}\right)
$$

Hence, the pair $\left(\varphi_{d, \mathscr{M}}, \omega_{d \mathscr{K}}\right) \in \mathscr{M} \times \mathscr{N}$ satisfies

$$
\begin{array}{r}
\left\langle\varphi_{d \mathscr{M}}^{\prime}, \psi^{\prime}\right\rangle+d^{-2}\left\langle\pi_{\mathscr{W}}\left(\varphi_{d, \mathscr{M}}-\omega_{d, \mathcal{H}}^{\prime}\right), \psi-v^{\prime}\right\rangle=\langle\mathrm{g}, v\rangle \\
\text { for all }(\psi, v) \in \mathscr{M} \times \mathscr{N} .
\end{array}
$$

\section{Mixed Finite Elements and Reduced Integration}

In this section we consider the special case of the discretization considered in the previous section in which, as in Sect. $4, \varphi_{d}$ and $\omega_{d}$ are approximated in $\mathscr{M}_{0}^{r}(\Delta)$. Thus we take $\mathscr{M}=\mathscr{N}=\mathscr{M}_{0}^{r}(\Delta)$ and $\mathscr{W}=\mathscr{M}_{1}^{r-1}(\Delta)$. Let $\left(\bar{\varphi}_{d \Delta}, \bar{\omega}_{d \Delta}, \bar{\zeta}_{d \Delta}\right) \in \mathscr{M} \times \mathscr{N} \times \mathscr{W}$ denote the Galerkin solution, the existence of which is guaranteed by Theorem 5.2.

Let $0<\xi_{1}<\xi_{2}<\ldots<\xi_{r}<1$ and $w_{1}, w_{2}, \ldots, w_{r}$ denote respectively the points and weights of the $r$ point Gauss quadrature rule on $[0,1]$. Define the approximate $L^{2}$ inner product

$$
\langle\varphi, \psi\rangle_{d}=\sum_{i=1}^{n}\left(x_{i}-x_{i-1}\right) \sum_{j=1}^{r} w_{j} \cdot(\varphi \psi)\left[x_{i-1}+\left(x_{i}-x_{i-1}\right) \xi_{j}\right] .
$$

for $\varphi, \psi \in \mathscr{M}_{-1}^{r}(\Delta)$. (Recall that $0=x_{0}<x_{1}<\ldots<x_{n}=1$ denote the knots of the partition $\Delta$.) A reduced integration finite element method is given by

find $\left(\tilde{\varphi}_{d \Delta}, \tilde{\omega}_{d A}\right) \in V^{r}(\Delta)$ such that

$$
\left\langle\tilde{\varphi}_{d \Delta}^{\prime}, \psi^{\prime}\right\rangle+d^{-2}\left\langle\tilde{\varphi}_{d A}-\tilde{\omega}_{d A}^{\prime}, \psi-v^{\prime}\right\rangle_{\Delta}=\langle g, v\rangle \quad \text { for all }(\psi, v) \in V^{r}(\Delta) . \quad\left(R_{d A}\right)
$$

It is easy to see that the problem $\left(R_{d A}\right)$ has a unique solution. The key observation of this section is that

$$
\left(\tilde{\varphi}_{d A}, \tilde{\omega}_{d A}\right)=\left(\bar{\varphi}_{d A}, \bar{\omega}_{d A}\right) \text { and } \bar{\zeta}_{d A}=d^{-2} \pi_{\cdot \mu_{-1}^{-1}(A)}\left(\tilde{\varphi}_{d A}-\tilde{\omega}_{d A}^{\prime}\right) \text {. }
$$

This is an example of a more general equivalence between certain mixed methods and particular reduced integration schemes [8], but we shall give the elementary proof here.

Comparing with (5.1) and (5.2) we see that to establish this claim it suffices to show that

$$
\left\langle\pi_{\mathscr{M}_{-1}-1(\Delta)} \rho, \mu\right\rangle=\langle\rho, \mu\rangle_{\Delta} \quad \text { for } \rho, \mu \in \mathscr{M}_{-1}^{r}(\Delta) .
$$

For $\rho \in \mathscr{M}_{-1}^{r}(\Delta)$ let $I_{\Delta} \rho \in \mathscr{M}_{-1}^{r-1}$ interpolate $\rho$ at the Gauss points. Then, for $\eta \in \mathscr{M}_{-1}^{r-1}(\Delta)$

$$
\left\langle I_{\Delta} \rho, \eta\right\rangle=\left\langle I_{\Delta} \rho, \eta\right\rangle_{\Delta}=\langle\rho, \eta\rangle_{A}=\langle\rho, \eta\rangle,
$$


so $I_{A} \rho=\pi_{\mu_{-1}^{r-1(\Delta)}} \rho$. Hence, for $\mu \in \mathscr{M}^{r}{ }_{-1}(\Delta)$,

$$
\left\langle\pi_{\mu_{-1}^{1}(A)} \rho, \mu\right\rangle=\left\langle I_{\Delta} \rho, \mu\right\rangle=\left\langle I_{\Delta} \rho, \mu\right\rangle_{\Delta}=\langle\rho, \mu\rangle_{\Delta},
$$

which establishes the claim.

The problem $\left(R_{d \Delta}\right)$ appears to be a minor modification of problem $\left(S_{d \Delta}\right)$. However, comparison of the following theorem with Theorem 4.1 shows that this modification has an extremely beneficial effect on the accuracy of approximation for small $d$.

Theorem 6.1. Let $\left(\varphi_{d}, \omega_{d}\right) \in V$ and $\left(\bar{\varphi}_{d \Delta}, \bar{\omega}_{d \Delta}\right) \in V^{r}(\Delta)$ solve $\left(S_{d}\right)$ and $\left(R_{d \Delta}\right)$ respectively. Denote by $\bar{\alpha}_{d A}=\varphi_{d}-\bar{\varphi}_{d A}$ and $\bar{\beta}_{d A}=\omega_{d}-\bar{\omega}_{d \Delta}$ the errors. Then there exists a constant $C$ independent of $d \in(0,1], g \in H^{r-1}$, and the partition $A$ such that

$$
\left\|\bar{\alpha}_{d \Delta}\right\|+\left\|\bar{\beta}_{d \Delta}\right\|+h\left(\left\|\bar{\alpha}_{d \Delta}^{\prime}\right\|+\left\|\bar{\beta}_{d \Delta}^{\prime}\right\|\right) \leqq C h^{r+1}\|g\|_{r-1} .
$$

Proof. Note that

$$
\inf _{\zeta \in M_{-1}-1(\Delta)}\left\|\zeta_{d}-\zeta\right\| \leqq C h^{r}\left\|\zeta_{d}\right\|_{r} \leqq C h^{r}\|g\|_{r-1}
$$

by standard approximation theory and a regularity result from Theorem 3.1 . Hence the estimates on $\bar{\alpha}_{d \Delta}^{\prime}$ and $\bar{\beta}_{d \Delta}^{\prime}$ follow directly from Theorem 5.2. Indeed this theorem gives us more. Putting $\bar{\zeta}_{d \Delta}=d^{-2} \pi_{M_{-!}^{r-1}(\Delta)}\left(\bar{\varphi}_{d \Delta}-\bar{\omega}_{d \Delta}^{\prime}\right)$ and $\bar{\gamma}_{d \Delta}-\zeta_{d}$ $-\bar{\zeta}_{d \Delta}$, the estimate

also holds.

$$
\left\|\bar{\gamma}_{d A}\right\| \leqq C h^{r}\|g\|_{r-1}
$$

To establish the $L^{2}$ estimates we use duality. Define $(\rho, \mu) \in V$ by

$$
B_{d}(\varphi, \omega ; \rho, \mu)=\left\langle\varphi, \bar{x}_{d A}\right\rangle+\left\langle\omega, \bar{\beta}_{d A}\right\rangle \quad \text { for all }(\varphi, \omega) \in V
$$

and set $\xi=d^{-2}\left(\rho-\mu^{\prime}\right)$. By Theorem 3.1,

$$
\|\rho\|_{2}+\|\mu\|_{2}+\|\xi\|_{1} \leqq C\left(\left\|\bar{\alpha}_{d \Delta}\right\|+\left\|\bar{\beta}_{d A}\right\|\right)
$$

Setting $\varphi=\bar{\alpha}_{d \Delta}, \omega=\bar{\beta}_{d \Delta}$ in (6.2) and using the definition of $\xi_{d}$ and the mixed finite element equations satisfied by $\left(\bar{\varphi}_{d \Delta}, \bar{\omega}_{d A}, \bar{\zeta}_{d \Delta}\right)$, we have

$$
\begin{aligned}
\left\|\bar{\alpha}_{d \Delta}\right\|^{2}+\left\|\bar{\beta}_{d \Delta}\right\|^{2}=\left\langle\bar{\alpha}_{d \Delta}^{\prime}, \rho^{\prime}\right\rangle+d^{-2}\left\langle\bar{\alpha}_{d \Delta}-\bar{\beta}_{d \Delta}^{\prime}, \rho-\mu^{\prime}\right\rangle \\
\quad=\left\langle\bar{\alpha}_{d \Delta}^{\prime}, \rho^{\prime}\right\rangle+\left\langle\bar{\gamma}_{d \Delta}, \rho-\mu^{\prime}\right\rangle+\left\langle\bar{\alpha}_{d \Delta}-\bar{\beta}_{d \Delta}^{\prime}-d^{2} \bar{\gamma}_{d \Delta}, \xi\right\rangle \\
\quad=\inf \left\langle\bar{\alpha}_{d \Delta}^{\prime}, \rho^{\prime}-\psi^{\prime}\right\rangle+\left\langle\bar{\gamma}_{d \Delta}, \rho-\psi-\mu^{\prime}+v^{\prime}\right\rangle+\left\langle\bar{\alpha}_{d \Delta}-\bar{\beta}_{d \Delta}^{\prime}-d^{2} \bar{\gamma}_{d \Delta}, \xi-\eta\right\rangle
\end{aligned}
$$

where the infimum is over all $(\psi, v) \in V^{r}(\Delta)$ and $\eta \in \mathscr{M}_{-1}^{r-1}(\Delta)$. Thus

as claimed.

$$
\begin{aligned}
\left\|\bar{\alpha}_{d \Delta}\right\|^{2}+\left\|\bar{\beta}_{d \Delta}\right\|^{2} & \leqq C h\left(\left\|\bar{\alpha}_{d d}^{\prime}\right\|+\left\|\bar{\beta}_{d d}^{\prime}\right\|+\left\|\bar{\gamma}_{d d}\right\|\right)\left(\left\|\rho^{\prime \prime}\right\|+\left\|\mu^{\prime \prime}\right\|+\left\|\xi^{\prime}\right\|\right) \\
& \leqq C h^{r+1}\|g\|_{r-1}\left(\left\|\bar{\alpha}_{d \Delta}\right\|+\left\|\bar{\beta}_{d \Delta}\right\|\right),
\end{aligned}
$$




\section{Proof of an Abstract Stability Theorem}

In this section we give the deferred proof of Theorem 5.1. Recalling the notation of that theorem, let also $y$ denote the orthogonal complement of $\mathscr{Z}^{\prime}$ in $\mathscr{V}$. We define the following linear operators:

$$
\begin{array}{ll}
A: \mathscr{Z} \rightarrow \mathscr{Z}, & \left\langle A z_{1}, z_{2}\right\rangle_{\mathscr{Y}}=a\left(z_{1}, z_{2}\right), \\
Q: \mathscr{Z} \rightarrow \mathscr{Y}, & \left\langle Q z_{1}, y_{1}\right\rangle_{\mathscr{Y}}=a\left(z_{1}, y_{1}\right), \\
R: \mathscr{Y} \rightarrow \mathscr{Y}, & \left\langle R y_{1}, y_{2}\right\rangle_{y}=a\left(y_{1}, y_{2}\right), \\
B: \mathscr{Y} \rightarrow \mathscr{W}, & \left\langle B y_{1}, w\right\rangle_{w}=b\left(y_{1}, w\right) .
\end{array}
$$

Here $z_{1}, z_{2} \in \mathscr{Z}, y_{1}, y_{2} \in \mathscr{Y}$, and $w \in \mathscr{W}$. Identifying $\mathscr{W}$ with $\mathscr{Z} \times \mathscr{Y}$, we also define a linear operator $\Pi_{d}: \mathscr{V} \times \mathscr{W} \rightarrow \mathscr{V} \times \mathscr{W}$ by

$$
\Pi_{d}(z, y, w)=\left(\begin{array}{ccc}
A & Q^{*} & 0 \\
Q & R & B^{*} \\
0 & B & -d^{2}
\end{array}\right)\left(\begin{array}{l}
z \\
y \\
w
\end{array}\right)
$$

for all $z \in \mathscr{Z}, y \in \mathscr{Y}, w \in \mathscr{W}$.

Using this notation we reinterpret the hypotheses and conclusion of Theorem 5.1. Hypothesis 2) is equivalent to the assertion that

$$
C_{1}\langle A z, z\rangle_{\Psi} \geqq\|z\|_{\sharp}^{2} \quad \text { for all } z \in \mathscr{Z} \text {. }
$$

This implies that $A$ is invertible and $\left\|A^{-1}\right\|_{\mathscr{Y}(\mathcal{Y}, \mathcal{Z})} \leqq C_{1}$. Let $P=R$ $-Q A^{-1} Q^{*}: \mathscr{Y} \rightarrow \mathscr{Y}$. Then

$$
\langle P y, y\rangle_{*}=a\left(y-A^{-1} Q^{*} y, y-A^{\cdot 1} Q^{*} y\right) \text { for all } y \in y^{\prime},
$$

so the first hypothesis assures that $P$ is positive semidefinite. The third hypothesis is equivalent to the invertibility of $B$ with $\left\|B^{-1}\right\|_{\left.\mathscr{S}_{(x)}, y_{y}\right)} \leqq C_{2}$. Letting $N_{d}=B^{*} B+d^{2} P$ it then follows that $N_{d}$ is positive definite and

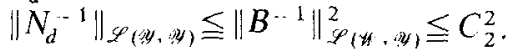

The assertion of the theorem is that $\Pi_{d}$ is invertible and $\left\|\Pi_{d}^{-1}\right\|_{\mathscr{L}\left(\boldsymbol{r}^{\prime} \times \mathscr{H}, * \times \mathbb{W}\right)} \leqq C$, with $C$ dependent only on $C_{1}, C_{2}$, and the norms of the linear operators $A, Q, R$, and $B$. We establish the theorem by exhibiting $\Pi_{d}^{-1}$.

$$
\begin{aligned}
& \Pi_{d}^{-1}(z, y, w) \\
& =\left[\begin{array}{ccc}
A^{-1}+d^{-2} A^{-1} Q^{*} N_{d}^{-1} Q A^{-1} & -d^{2} A^{-1} Q^{*} N_{d}^{-1} & -A^{-1} Q^{*} N_{d}^{-1} B^{*} \\
-d^{2} N_{d}^{-1} Q A^{-1} & d^{2} N_{d}^{-1} & N_{d}^{-1} B^{*} \\
-B N_{d}^{-1} Q A^{-1} & B N_{d}^{-1} & -B N_{d}^{-1} P B^{-1}
\end{array}\right]\left[\begin{array}{l}
z \\
y \\
w
\end{array}\right]
\end{aligned}
$$

To verify this formula one simply multiplies the matrices in (7.1) and (7.2) and performs some algebraic manipulation. 

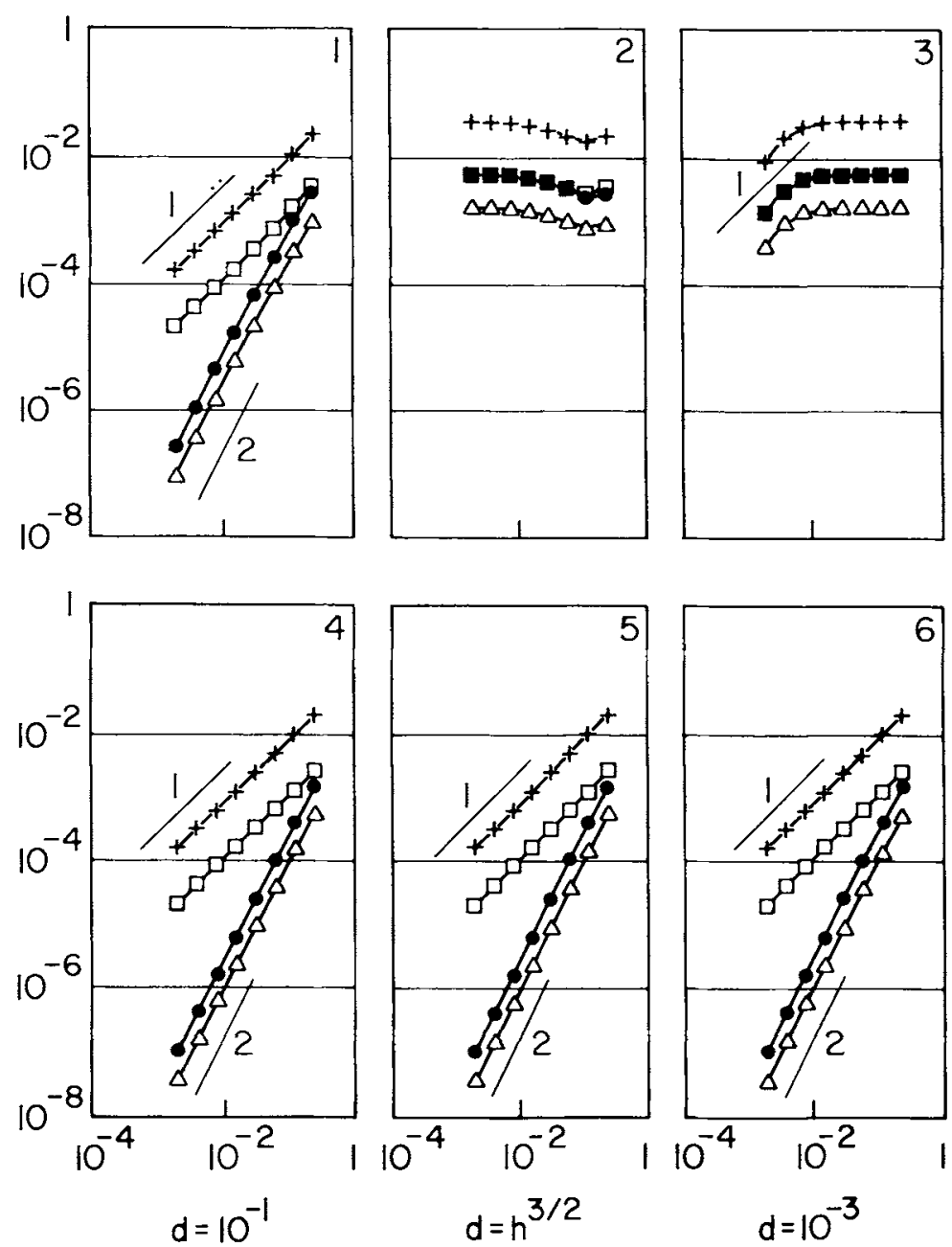

Fig. 1. Graphs 1-3: Standard finite element method. Graphs 4-6: Reduced integration finite element method. In Graphs 1 and $4, d=10^{-1}$, in Graphs 2 and $5, d=h^{3}$; in Graphs 3 and 6 , $d=10^{-3}$.

Error in linear finite element approximations plotted as a function of $h$.

- $L^{2}$ norm of error in $\varphi_{a}$

$\triangle L^{2}$ norm of error in $\omega_{d}$

$+L^{2}$ norm of error in $\varphi_{d}^{\prime}$

$\square L^{2}$ norm of error in $\omega_{d}^{\prime}$

Numbered line segments are drawn at the marked slopes for comparison

Remark. Formula (7.2) is valid for $d=0$ and may be used to compare $\Pi_{d}^{-1}$ to $\Pi_{0}^{-1}$. One easily checks that $\left\|\Pi_{d}^{-1}-\Pi_{0}^{-1}\right\|_{\mathscr{L}(\mathscr{W} \times \mathscr{W}, \mathscr{W} \times \mathscr{W})} \leqq C d^{2}$, which furnishes an elementary proof of the major theorem of [2] under slightly more general hypotheses. This justifies the use of the method $\left(R_{d A}\right)$ as a penalisation method for the biharmonic limiting problem. 

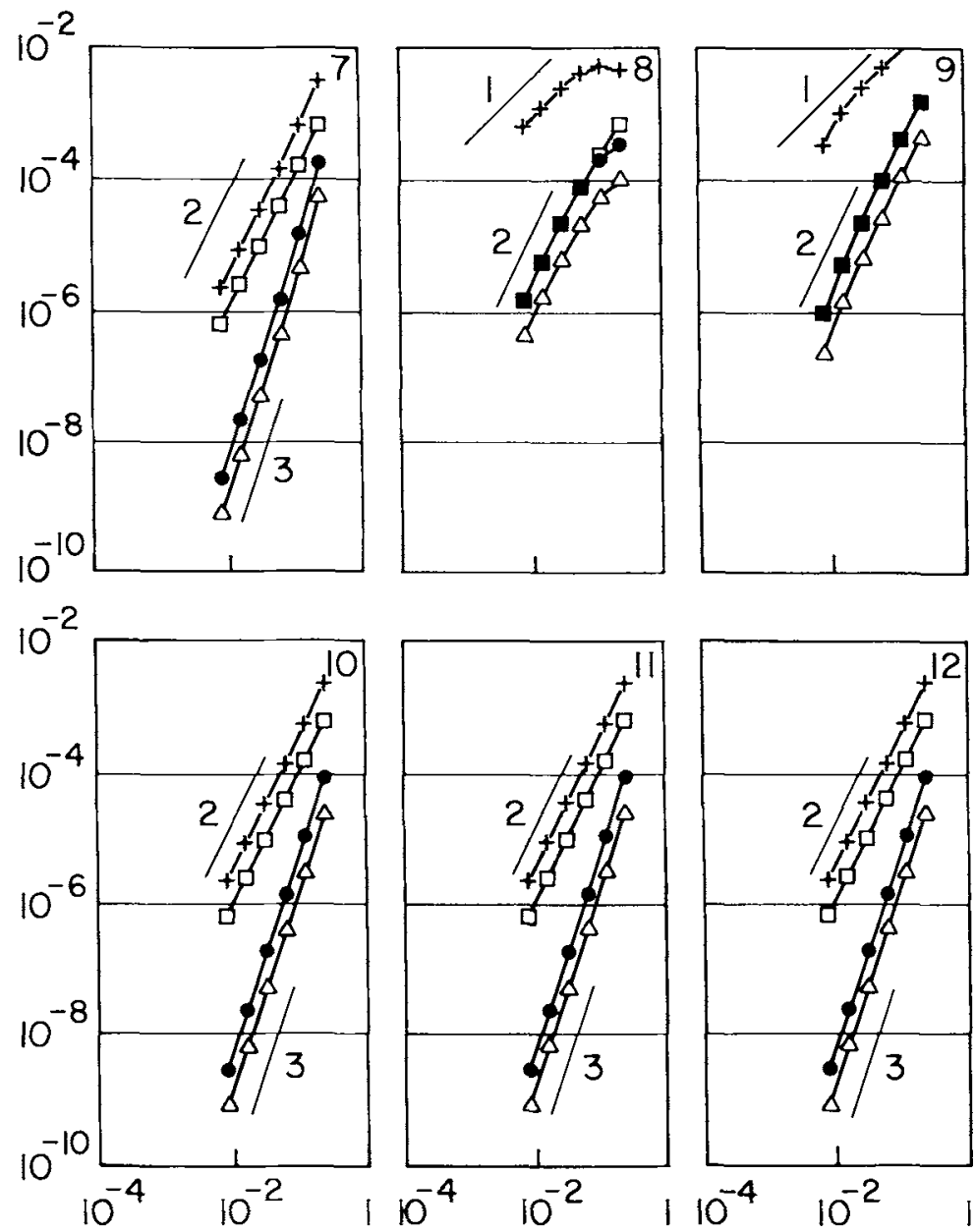

$$
\mathrm{d}=10^{-1}
$$

$$
d=h^{2}
$$$$
d=10^{-3}
$$

Fig. 2. Graphs 7-9: Standard finite element method. Graphs 10-12: Reduced integration finite element method. In Graphs 7 and $10, d=10^{-1}$; Graphs 8 and $11, d=h^{2}$; in Graphs 9 and 12, $d=10^{-3}$.

Error in quadratic finite element approximation plotted as a function of $h$.

- $L^{2}$ norm of error in $\varphi_{d}$

$\Delta L^{2}$ norm of error in $\omega_{d}$

$+L^{2}$ norm of error in $\varphi_{d}^{\prime}$

$\square L^{2}$ norm of error in $\omega_{d}^{\prime}$

Numbered line segments are drawn at the marked slopes for comparison

\section{Numerical Results}

In this section we give the results of computations of the solutions to the standard finite element equations $\left(S_{d A}\right)$ and the reduced integration equations $\left(R_{d A}\right)$. For simplicity the mesh $\Delta$ was taken to be uniform and the loading 
function $g$ to be identically one in all cases. All computations were performed on a UNIVAC 1108 computer using 60 bit double precision arithmetic.

Two sets of computations are presented, each being exhibited in six graphs. The first set of computations were performed with linear finite elements, the second set with quadratics.

In each graph the $L^{2}$ norm of the error in each of the four quantities $\varphi_{d}$, $\omega_{d}, \varphi_{d}^{\prime}$, and $\omega_{d}^{\prime}$ is graphed as a function of $h$. The values of $h$ used were $2^{-k}$ with $k=2,3, \ldots, 9$ in the linear case, $k=2,3, \ldots, 7$ in the quadratic case. Both axes have been transformed logarithmically so that the slope of the error curves gives the apparent rate of convergence as $h$ tends to zero. Absolute errors are shown. The exact solution depends on $d$, but for the computations here none of the quantities $\left\|\varphi_{d}\right\|,\left\|\omega_{d}\right\|,\left\|\varphi_{d}^{\prime}\right\|,\left\|\omega_{d}^{\prime}\right\|$ differs by more than a factor of 2 from its value when $d=0.1$. These values are:

$$
\begin{aligned}
\left\|\varphi_{0.1}\right\| & =5.8 \times 10^{-3}, \\
\left\|\omega_{0.1}\right\| & =2.6 \times 10^{-3}, \\
\left\|\varphi_{0.1}^{\prime}\right\| & =3.7 \times 10^{-2}, \\
\left\|\omega_{0.1}^{\prime}\right\| & =8.3 \times 10^{-3}
\end{aligned}
$$

The errors for the standard linear finite element method are shown in Graphs 1-3. For the first graph $d=0.1$ and convergence at the expected rates (cf. the first line of Table 1 ) is evident. In the second graph $d$ varies with $h(d$ $=h^{\frac{3}{2}}$ ) and the lack of any uniform approximation is clear. In fact the finite element solution does not converge to the true solution at all in this case, as was shown in the proof of Theorem 4.2, and is clear from the graph. Possibly more surprising is the third graph in which $d$ is held constant but small: $d$ $=0.001$. Although the approximation is known to converge at the optimal rate asymptotically, no convergence is apparent until a very high level of discretization is achieved. This highlights the importance of uniform approximation.

Graphs 4-6 show the results of solving the same problems by linear finite elements with reduced integration. The uniformly optimal order convergence predicted by the theory is evident. Indeed the error in approximation is seen to be nearly independent of $d$.

Graphs 7-9 pertain to the standard finite element method with quadratic elements in the cases $d=0.1, d=h^{2}$, and $d=0.001$ respectively. The final three plots display the analagous information for the quadratic finite element method with the integrations reduced to two point composite Gaussian quadratures as explained in Sect. 6. Again, that uniformly optimal order convergence is achieved by the latter method is apparent even for $h$ quite large $(h>0.1)$. On the other hand the suboptimal rates given in Table 1 are exhibited by the standard method in the eighth graph. Moreover the ninth graph indicates that when $d$ is small but constant the behavior of the standard finite element solution is best described not by the ultimate asymptotic rates, but by the convergence rates which hold uniformly in $d$. 
Acknowledgements. The author is grateful to Ivo Babuška for suggesting this area of investigation and to Babuška and John Osborn for many useful discussions.

The computations presented were performed with funds provided by the Computer Science Center of the University of Maryland.

This work was supported in part by the General Research Board of the University of Maryland.

\section{References}

1. Babuška, I., Aziz, A.K.: Survey lectures on the finite element method. In: The mathematical foundations of the finite element method with applications to partial differential equations (A.K. Aziz, ed.), pp. 5-359. New York: Academic Press 1973

2. Bercovier, M.: Perturbation of mixed variational problems. Application to mixed finite element methods. Rev. Française Automat. Informat. Recherche Opérationnelle Sér. Rouge Anal. Numér. 12, 211-236 (1978)

3. Brezzi, F.: On the existence, uniqueness and approximation of saddle point problems arising from Lagrangian multipliers. Rev. Française Automat. Informat. Recherche Opérationnelle 8 R-2, 129-151 (1974)

4. Falk. R., Osborn, J.: Error estimates for mixed methods. Rev. Française Automat. Informat. Recherche Opérationnelle Sér. Rouge Anal. Numér. (in press 1981)

5. Fried, I.: Finite element analysis of incompressible materal by residual energy balancing. Internat. J. Solids and Structures 10, 993-1002 (1974)

6. Hughes, T.J.R., Cohen, M., Haroun, M.: Reduced and selective integration techniques in the finite element analysis of plates. Nuclear Engrg. Design 46, 203-222 (1978)

7. Hughes, T.J.R., Taylor, R.L., Kanoknukulchai, W.: A simple and efficient finite element for plate bending. Internat. J. Numer. Methods Engrg. 11, 1529-1543 (1977)

8. Malkus, D.S., Hughes, T.J.R.: Mixed finite element methods-reduced and selective integration techniques: a unification of concepts. Comput. Methods Appl. Mech. Engrg. 15, 63-81 (1978)

9. Pawsey, S.F., Clough, R.W.: Improved numerical integration of thick shell finite elements. Internat. J. Numer. Methods Engrg. 3, 545-586 (1971)

10. Pugh, E.D.L., Hinton, E., Zienkiewicz, O.C.: A study of quadrilateral plate bending elements with reduced integration. Internat. J. Numer. Methods Engrg. 12, 1059-1079 (1978)

11. Timoshenko, S.P.: On the correction for shear of the differential equation for transverse vibrations of prismatic bars. Philos. Mag. Ser. 6, 41, 744-746 (1921)

12. Zienkiewicz, O.C.. Hinton, E.: Reduced integration, function smoothing and non-conformity in finite element analysis (with special reference to thick plates). J. Franklin Inst. 302, 443-461 (1976)

13. Zienkiewicz, O.C., Taylor, R.L.. Too, J.M.: Reduced integration techniques in general analysis of plates and shells. Internat. J. Numer. Methods Engrg. 5, 275-290 (1971) 